\section{UMA ANÁLISE SOBRE O ACOMPANHAMENTO DO NÚMERO DE ÓBITOS PROVOCADOS PELA COVID-19}

DOI: 10.37702/2175-957X.COBENGE.2021.3564

EUCLIDES HONÓRIO DE ARAÚJO - euclides@ufu.br

UFU

Rua Alexandrino Santos Lima 150

38408-082 - Uberlândia - MG

Resumo: Neste trabalho é apresentado um estudo sobre a COVID-19 no país, tendo por base um Quadro Resumo que mostra a evolução no número de óbitos. Em seguida é discutido, com base em parâmetros como, o Tempo de Duplicação e a Variação Mensal, a situação do país, de cada estado da federação, e da cidade de Uberlândia-MG.

Palavras-chave: Óbitos pela Covid-19, Corona vírus no Brasil. 


\section{UMA ANÁLISE SOBRE O ACOMPANHAMENTO DO NÚMERO DE ÓBITOS PROVOCADO PELA COVID-19 INTRODUÇÃO}

Dentro do escopo dos temas transversais apresentamos como contribuição ao ensino da engenharia, uma modelagem que mostra como que um profissional desta área pode acompanhar o desenrolar de um fenômeno de caráter sanitário, com base na correlação dos dados disponíveis.

Neste trabalho é apresentado uma metodologia, ressaltada por Lacerda (2020), para acompanhamento do número de óbitos da pandemia de Coronavirus, utilizando os dados desde o início do surto da doença. São acompanhados os dados nacionais assim como de quatro estados a saber: São Paulo, Rio de Janeiro, Pernambuco e Minas Gerais. Estudase também os dados da cidade de Uberlândia-MG.

É apresentado um resumo que aparece no Quadro 1 onde as principais informações são apresentadas, a partir dos dados divulgados diariamente pelo Conselho Nacional de Secretários da Saúde - CONASS.

Além do Quadro 1 são apresentadas seis figuras que mostram, ao longo do tempo, o desenrolar da pandemia com os números de óbitos.

\section{METODOLOGIA EMPREGADA NO ESTUDO}

Para a obtenção dos resultados apresentados foram utilizados os dados para a população, encontrados no IBGE (2020, 2021), assim como os dados apresentados diariamente pelo CONASS $(2020,2021)$.

\subsection{Tempo de duplicação - Td, do número de óbitos}

Este parâmetro é muito importante pois dá uma medida de quanto evoluiu uma cidade ou estado no sentido de chegar mais próximo do final da pandemia. Pandemia.

O valor do Td é expresso em dias e explicita há quanto tempo o valor no número de óbitos era a metade do atual.

Quando se tem um aumento considerável no número de óbitos isto é imediatamente refletido no abaixamento do $\mathrm{Td}$ da cidade, do estado ou do país.

\subsection{Variação mensal no número de óbitos}

Este segundo parâmetro também expressa em que medida o número de óbitos aumenta. Para se obter este parâmetro é feito o cálculo de quanto foi a variação em um período de trinta dias.

\subsection{Variação diária no número de óbitos}

De forma semelhante o cálculo da variação diária no número de óbitos é obtido comparando-se os dados de acréscimos diários no número de óbitos para cada estado, para o país e para a cidade. 


\section{PARÂMETROS SECUNDÁRIOS UTILIZADOS NO ESTUDO}

Alguns parâmetros que mostram os resultados obtidos para o acompanhamento da pandemia foram obtidos utilizando os dados das populações dos estados e também dos dados acumulativos do número de óbitos diários.

\subsection{Número de óbitos por milhão de habitantes}

O número de óbitos por milhão de habitantes é a divisão do número acumulado diariamente, pela população de cada estado. Os valores populacionais são mantidos constantes durante o estudo e não comprometem os resultados obtidos.

\subsection{Porcentagem de óbitos por estado}

A porcentagem de óbitos por estado é obtida pela divisão do número diário acumulado de óbitos pela população de cada estado.

\section{$4 \quad$ APRESENTAÇÃO DOS RESULTADOS}

Passamos agora a apresentar os resultados obtidos após a metodologia empregada ser apresentada. Com a pandemia em andamento apresentamos os dados da semana encerrada em 07.5.21, mas os dados são atualizados diariamente.

\subsection{Quadro do Resumo geral dos resultados}

Na sequência apresentamos o Quadro 1 que explicita o estudo.

O Quadro 1 apresenta doze colunas que mostram respectivamente: a) os entes estudados, b) a população dos entes, c) o número de óbitos trinta dias atrás, d) o número de óbitos na data estudada, e) a variação ocorrida no período de um mês, f) a proporção de óbitos por milhão de habitantes para cada ente da federação, g) o Tempo de duplicação - Td, para o ente, $\mathbf{h}$ ) o aumento diário no número de óbitos, i) o número de óbitos no dia anterior à data em estudo, j) a porcentagem de óbitos para cada ente, $\mathbf{k}$ ) o número de óbitos na data estudada, e I) a variação semanal no número Td. 
Quadro 1 - Resumo da situação diária da pandemia com a relação aos óbitos

\begin{tabular}{|c|c|c|c|c|c|c|c|c|c|c|c|c|}
\hline \multirow[t]{2}{*}{ Estado } & \multicolumn{2}{|r|}{ População } & \multirow{2}{*}{$\begin{array}{c}\mathrm{N}^{0} \text { de Deaths } \\
07.4 .21\end{array}$} & \multicolumn{2}{|c|}{$N^{\circ}$ de Deaths } & & \multirow{2}{*}{$\begin{array}{l}\text { Td* atual } \\
\text { dias }\end{array}$} & \multirow{2}{*}{$\begin{array}{l}\text { Aumento } \\
\text { diário \% }\end{array}$} & \multirow{2}{*}{\multicolumn{2}{|c|}{$\begin{array}{c}N^{*} \text { de Dea Deaths } \\
06.5 .21 \%\end{array}$}} & \multirow{2}{*}{\begin{tabular}{|l|} 
Daily \\
Deaths
\end{tabular}} & \multirow{2}{*}{$\begin{array}{l}\text { Delta } \\
\mathrm{Td}(1)\end{array}$} \\
\hline & & M & & 07.5 .21 & Variação & Deaths/M hab & & & & & & \\
\hline & & & & & & & & & & & & \\
\hline Acre & & 0,89 & 1318 & 1575 & 1,19499 & 1769,662921 & 130 & 0,446429 & 1568 & 0,375793 & 7 & -1 \\
\hline Alagoas & & 3,35 & 3710 & 4347 & 1,1717 & 1297,61194 & 202 & 0,4158 & 4329 & 1,037188 & 18 & -4 \\
\hline Amapá & & 0,86 & 1356 & 1578 & 1,16372 & 1834,883721 & 167 & 0,317864 & 1573 & 0,376509 & 5 & -2 \\
\hline \multicolumn{2}{|l|}{ Amazonas } & 4,21 & 12177 & 12716 & 1,04426 & 3020,427553 & 109 & 0,070827 & 12707 & 3,034019 & 9 & 7 \\
\hline Bahia & & 14,93 & 16107 & 19071 & 1,18402 & 1277,361018 & 113 & 0,479452 & 18980 & 4,550313 & 91 & -3 \\
\hline Ceará & & 9,19 & 14884 & 18265 & 1,22716 & 1987,486398 & 208 & 0,53944 & 18167 & 4,358003 & 98 & -20 \\
\hline \multicolumn{2}{|l|}{ D. Federal } & 3,06 & 6532 & 8056 & 1,23331 & 2632,679739 & 149 & 0,373785 & 8026 & 1,92215 & 30 & -8 \\
\hline E. Santo & & 4,06 & 7937 & 9831 & 1,23863 & 2421,428571 & 133 & 0,480376 & 9784 & 2,345663 & 47 & 2 \\
\hline Goiás & & 7,11 & 12343 & 15544 & 1,25934 & 2186,216596 & 87 & 0,497834 & 15467 & 3,708776 & 77 & -1 \\
\hline \multicolumn{2}{|l|}{ Maranhão } & 7,11 & 6369 & 7503 & 1,17805 & 1055,274262 & 219 & 0,401445 & 7473 & 1,790205 & 30 & -3 \\
\hline \multicolumn{2}{|l|}{ Mato Groso } & 3,53 & 8184 & 9907 & 1,21053 & 2806,515581 & 101 & 0,303736 & 9877 & 2,363796 & 30 & 1 \\
\hline & 2,81 & 4630 & 5961 & 1,28747 & 2121,352313 & 91 & 0,404245 & 5937 & 1,422286 & 24 & -2 \\
\hline \multicolumn{2}{|l|}{ M Gerais } & 21,29 & 26303 & 35424 & 1,34677 & 1663,879756 & 75 & 0,736528 & 35165 & 8,452116 & 259 & 0 \\
\hline \multicolumn{2}{|l|}{ Pará } & 8,69 & 10880 & 13453 & 1,23649 & 1548,101266 & 193 & 0,462998 & 13391 & 3,209867 & 62 & -25 \\
\hline \multicolumn{2}{|l|}{ Paraíba } & 4,04 & 6050 & 6996 & 1,15636 & 1731,683168 & 141 & 0,459506 & 6964 & 1,669236 & 32 & -1 \\
\hline \multicolumn{2}{|l|}{ Paraná } & 11,52 & 18118 & 23623 & 1,30384 & 2050,607639 & 66 & 0,553356 & 23493 & 5,636414 & 130 & -1 \\
\hline \multicolumn{2}{|c|}{ Pernambuco } & 9,62 & 12541 & 14434 & 1,15094 & 1500,4158 & 263 & 0,340633 & 14385 & 3,443932 & 49 & 2 \\
\hline Piaui & & 3,28 & 4341 & 5296 & 1,22 & 1614,634146 & 155 & 0,684411 & 5260 & 1,263618 & 36 & -8 \\
\hline \multicolumn{2}{|c|}{ Rio de Janeiro } & 17,37 & 38282 & 46171 & 1,20608 & 2658,088659 & 153 & 0,559742 & 45914 & 11,01633 & 257 & -3 \\
\hline \multicolumn{2}{|l|}{ Rio G Sul } & 11,42 & 21261 & 25807 & 1,21382 & 2259,807356 & 65 & 0,54153 & 25668 & 6,157513 & 139 & 5 \\
\hline \multicolumn{2}{|l|}{ Rio G Norte } & 3,53 & 4786 & 5619 & 1,17405 & 1591,784703 & 144 & 0,357207 & 5599 & 1,340685 & 20 & -1 \\
\hline \multicolumn{2}{|l|}{ Rondônia } & 1,8 & 4419 & 5311 & 1,20186 & 2950,555556 & 77 & 0,568074 & 5281 & 1,267197 & 30 & 5 \\
\hline \multicolumn{2}{|l|}{ Roraima } & 0,63 & 1367 & 1534 & 1,12217 & 2434,920635 & 141 & 0,065232 & 1533 & 0,36601 & 1 & 1 \\
\hline \multicolumn{2}{|l|}{ S Catarina } & 7,25 & 11613 & 13992 & 1,20486 & 1929,931034 & 74 & 0,437872 & 13931 & 3,338471 & 61 & 1 \\
\hline \multicolumn{2}{|l|}{ S Paulo } & 46,29 & 79443 & 99989 & 1,25863 & 2160,056168 & 109 & 0,586484 & 99406 & 23,85723 & 583 & -3 \\
\hline Sergipe & & 2,32 & 3668 & 4469 & 1,21838 & 1926,293103 & 182 & 0,630489 & 4441 & 1,066297 & 28 & -11 \\
\hline Tocantins & & 1,59 & 2157 & 2642 & 1,22485 & 1661,63522 & 108 & 0,456274 & 2630 & 0,630377 & 12 & -1 \\
\hline BR & & 211,75 & 340776 & 419114 & 1,22988 & 1979,286895 & 111 & 0,519248 & 416949 & 100 & 2165 & 0 \\
\hline Udi & & 0,7 & 1890 & 2211 & 1,16984 & 3158,571429 & 66 & 0,454339 & 2201 & 0,527541 & 10 & 5 \\
\hline * & $d \mathrm{~T}$ & empo de du & ração no No d & & httos://vi & zww.conass.o & & & & & & \\
\hline 1- Variação & Sen & manal do $\mathrm{Tc}$ & & & & & & & & & & \\
\hline
\end{tabular}

\subsection{Figuras ilustrativas do estudo}

Agora passamos a apresentar os gráficos que representam a evolução da Pandemia em termos de número de óbitos para cada ente da União.

Em primeiro lugar apresentamos os dados para a União com data da semana do envio do artigo.

A Figura 1 representa a evolução do número de óbitos ao longo do tempo e com os números de óbitos representados na forma do logaritmo neperiano, para tornar a apresentação mais compacta.

O que se pode notar em termos nacionais é que a pandemia apresenta duas fases distintas, uma primeira onda que se prolonga até cerca de um ano, seguida de uma segunda onda, a qual estamos vivenciando e, felizmente esta segunda onda não apresenta uma aceleração tão pronunciada como ocorreu na anterior.

Mais recentemente o que tem ocorrido é uma desaceleração do crescimento no número de óbitos que é discutido na Sessão 5.

Em seguida apresentamos a Figura 2 que mostra os dados para o Estado de São Paulo. O Estado de São Paulo como pode ser observado no Quadro 1 é responsável por cerca de $24 \%$ do número de óbitos da União, assim pode-se afirmar que o Estado de SP tem grande influência na forma da curva apresentada na Figura 1 para a União, ou seja as duas curvas são semelhantes nas suas conformações. 
Figura 1 - Dados da evolução da pandemia, ao longo do tempo, para a União.

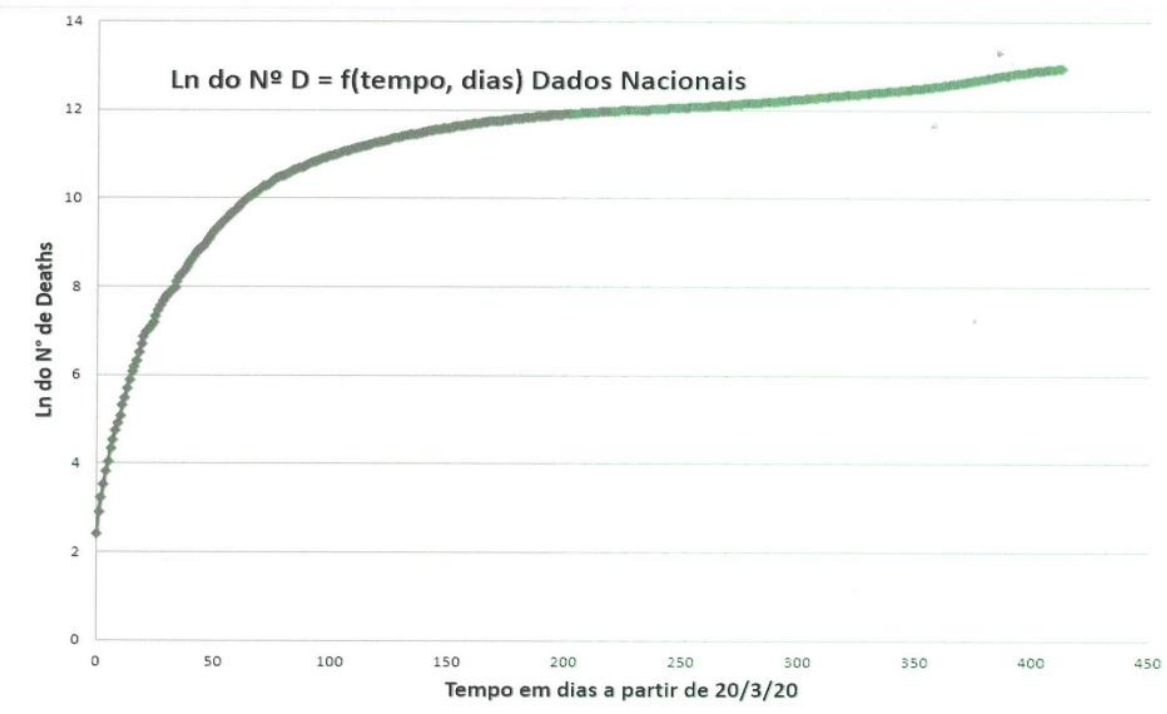

A Figura 3 mostra a evolução da pandemia ao longo do tempo para o Estado de MG. Como o segundo estado mais populoso, apresenta o terceiro maior número de óbitos com cerca de $8,5 \%$ do total nacional.

A curva do Estado de MG é ligeiramente diferente, das duas anteriores, especialmente até por volta do centésimo dia da pandemia, quando ainda apresentava uma velocidade de crescimento menor, nesta oportunidade o Estado de MG era o estado com a menor taxa de óbitos por milhão de habitantes, isto não mais ocorre, estando agora MG em sétimo lugar neste quesito.

A Figura 4 apresenta o desenrolar da pandemia para o Estado do RJ, esta curva é ligeiramente diferente das anteriores, pois a segunda onda só aparece após o ducentésimo dia e assim mesmo de forma mais suavizada.

O Estado do RJ apesar de contar com a terceira maior população é responsável pelo segundo lugar na porcentagem de óbitos, apresentado cerca de $11,0 \%$ do total da União. 
Figura 2 - Dados da evolução da pandemia, ao longo do tempo, para o Estado de SP.

Ln do no de óbitos em função do tempo (dias) Estado de SP

14

12

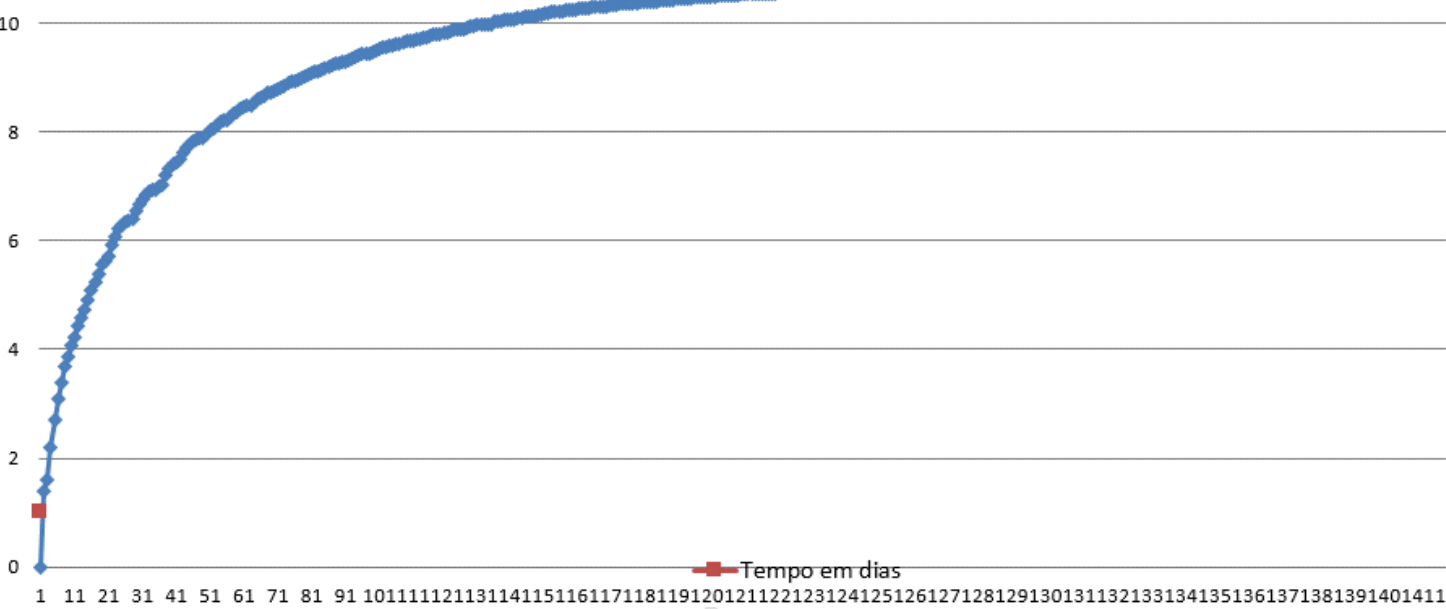

Figura 3 - Dados da evolução da pandemia, ao longo do tempo, para o Estado de MG.

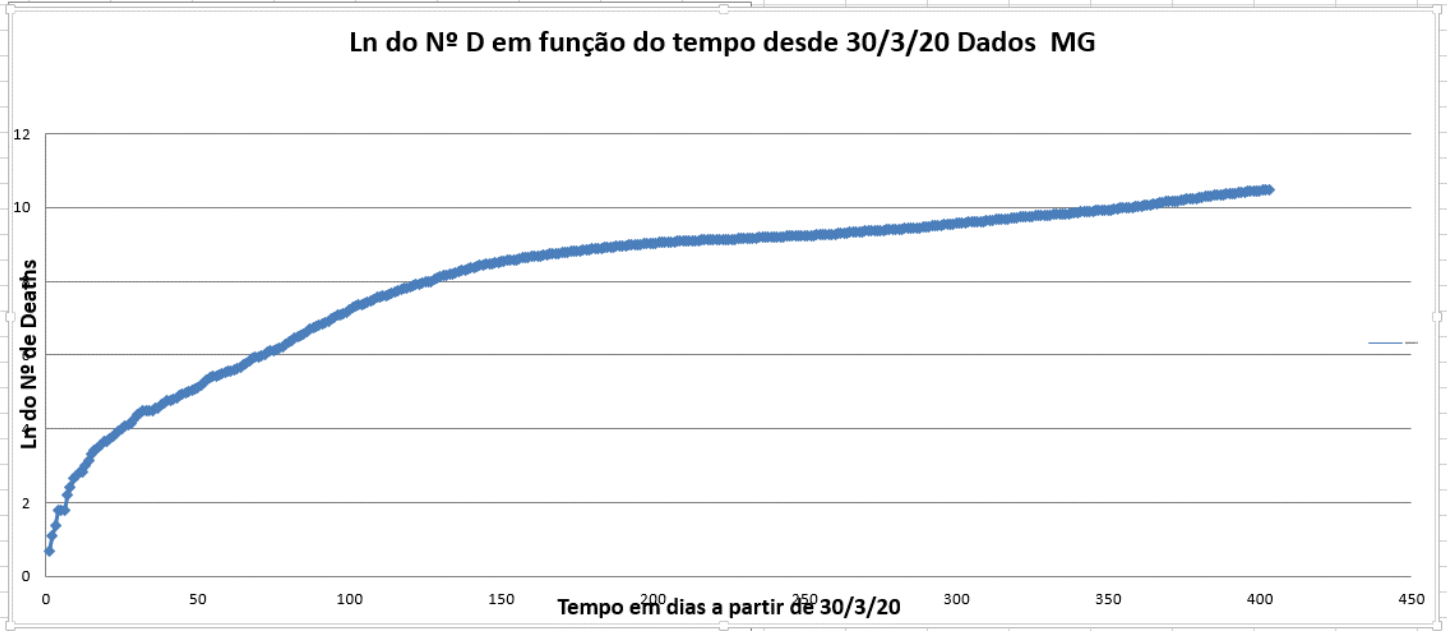

Em seguida apresentamos a Figura 5 da evolução da pandemia para o Estado de Pernambuco.

O Estado de PE apresenta até o momento o melhor desempenho, fato que pode indicar um final da pandemia mais próximo, isto é explicado pelo fato da velocidade de crescimento no número de óbitos ter sido sempre abaixo do crescimento médio no número de óbitos apresentado pela União.

Na sequência apresentamos a Figura 6 que mostra a da evolução da pandemia para a cidade Uberlândia-MG. 
Com estas seis figuras procura-se apresentar uma visão do comportamento da pandemia com um número razoável de estados representativos.

\section{DISCUSSÃO DOS RESULTADOS}

Passamos agora a discutir os pontos mais importantes que merecem ser abordados para aclarar a evolução da pandemia até o dia sete de maio de 2021, mas esta metodologia de abordagem pode ser empregada em qualquer momento.

Neste tópico serão utilizados os dados presentes no Quadro 1.

Figura 4 - Dados da evolução da pandemia, ao longo do tempo, para o Estado de RJ.

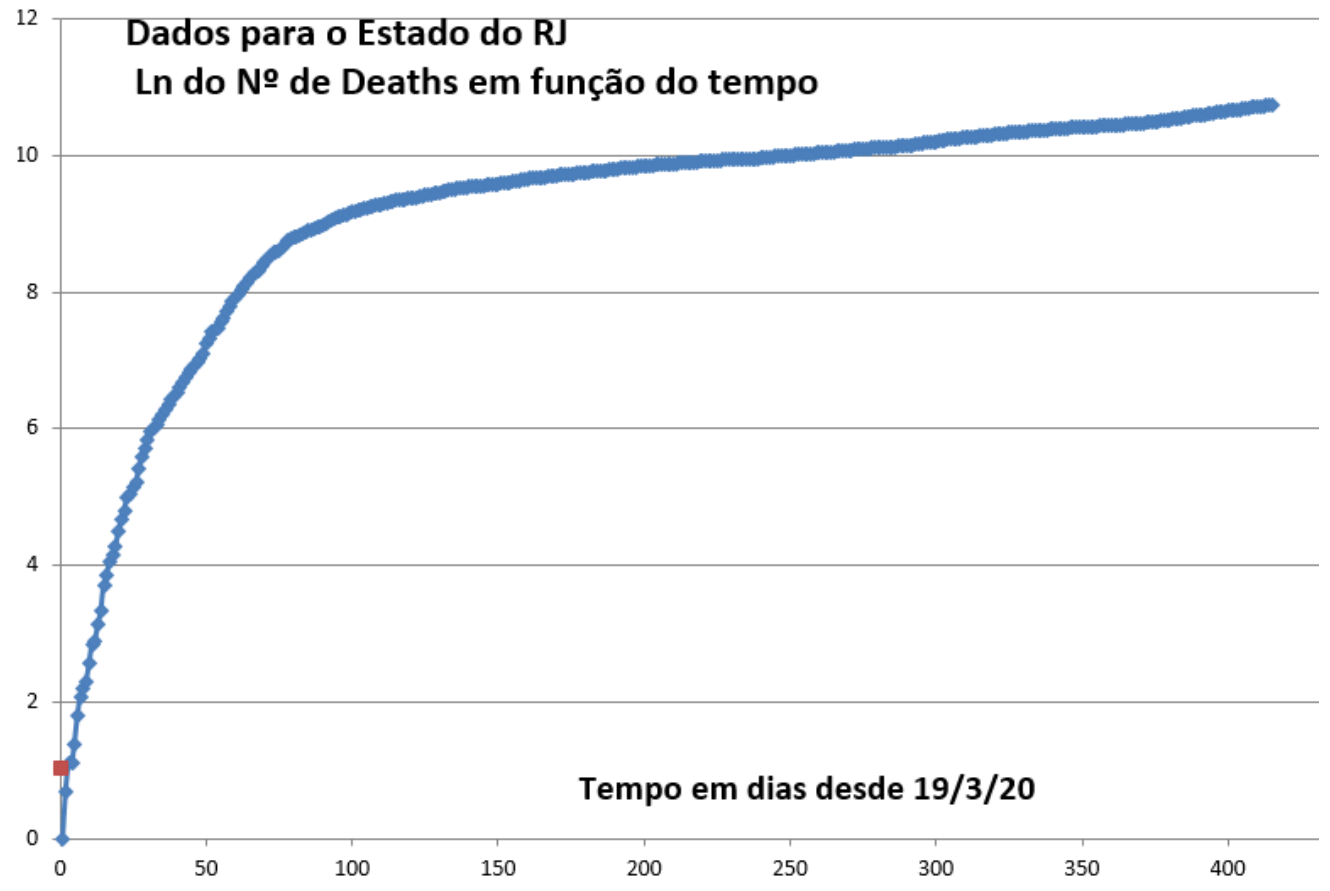

5.1 Análise segundo o tempo de duplicação Td

Os estados foram agrupados em dois blocos, o primeiro para aqueles com números de Td acima da média nacional e os outros que apresentam Td abaixo do Td médio nacional que no momento é igual a 111 dias, como pode ser verificado no Quadro 1.

\section{Estados com Td acima de 111 dias}

Td.

Neste bloco apresentamos os estados pela ordem decrescente dos seus números

PE (263); MA (219); CE (208); AL (202); PA (193); SE (182); AP (167); PI (155); DF (149); RJ (153); RN (144); PB (141); RR (141); ES (133); AC (130) e BA (113). 
Figura 5 - Dados da evolução da pandemia, ao longo do tempo, para o Estado de PE.

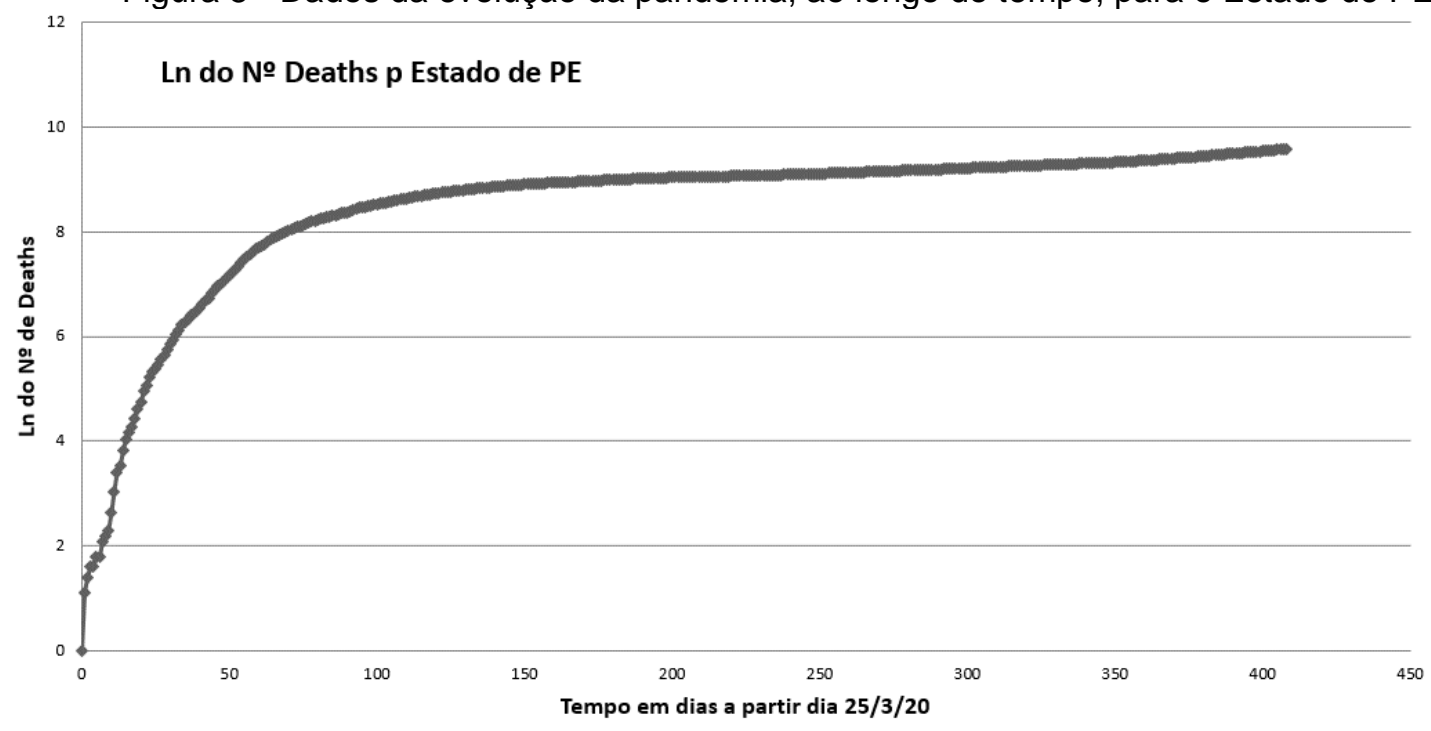

Figura 6 - Dados da evolução da pandemia para a cidade de Uberlândia-MG.

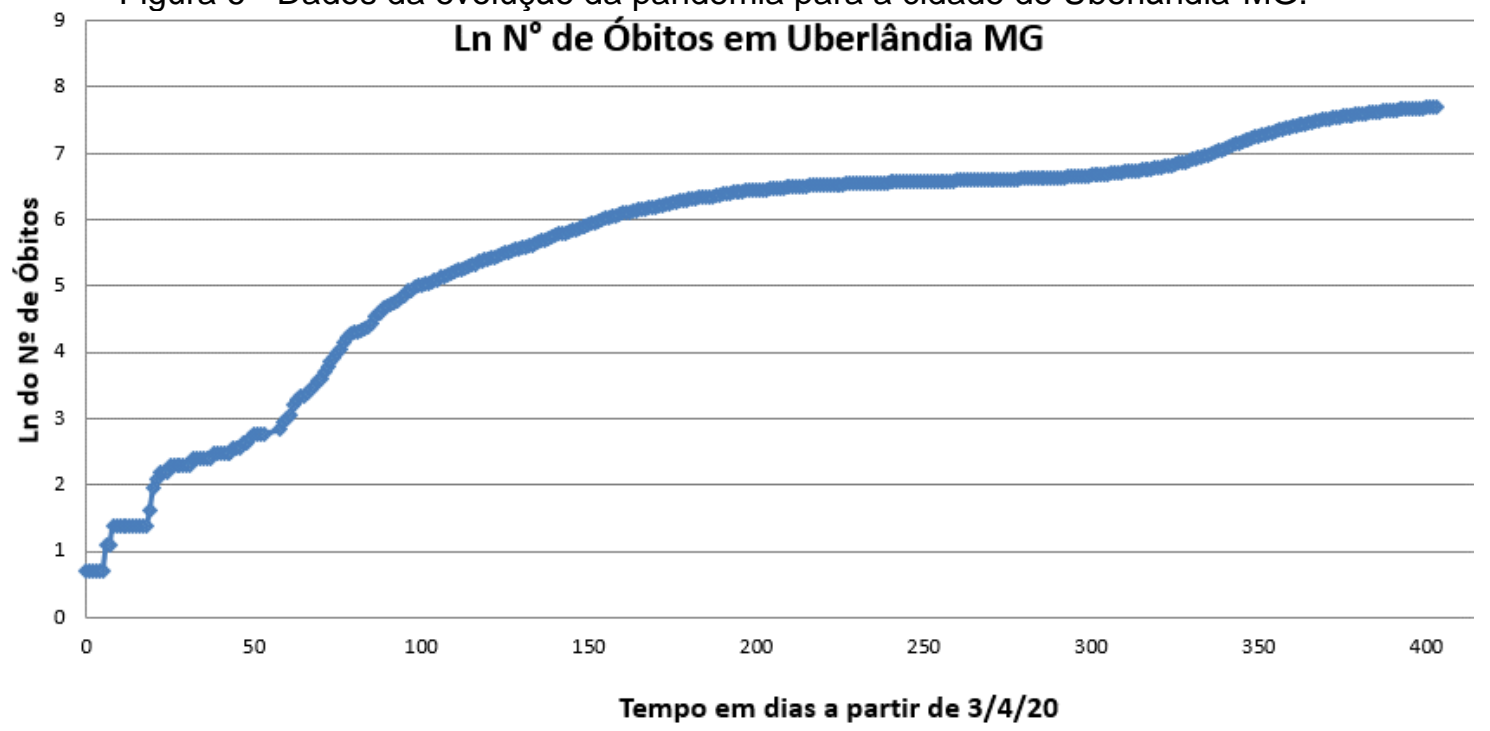

Variações no Td de valor acima de 111, de 30.4.21 a 07.5.21.

PE (2); ES (2); RR (1); PB (-1); RN (-1); AC (-1); AP (-2); MA (-3); SP (-3); RJ (-3); BA (-3); AL (-4); PI (-8); DF (-8); SE (-11); CE (-20) e PA (-25).

Em primeiro lugar devemos observar que o número Td nacional permaneceu inalterado, indicando uma situação que aponta para um arrefecimento relativo da pandemia.

Considerando esta manutenção do número Td nacional, observamos que as únicas unidades que tiveram os seus números Td aumentados foram: PE, ES e RR. Todos os outros estados tiveram variações negativas no $\mathrm{Td}$.

Destaco que os Estados que tiveram as maiores reduções nos seus Tds, foram: SE (-11); CE (-20) e PA (-25).

O Estado do MA continua com o menor número de óbitos por milhão de habitantes com o valor de 1.055 .

O Estado de PE, com uma das melhores curvas assintóticas, está com uma média de óbitos abaixo da média nacional por milhão de habitantes, ficando com 1.500 óbitos/M 
de habitantes, além disso, o estado apresenta uma taxa média de crescimento diário, no número de óbitos, de 0,397\%, enquanto a média diária de crescimento nacional, no mesmo período foi $0,531 \%$.

\section{Estados com Td abaixo de 111 dias}

SP (109); AM (109); TO (108); MT (101); MS (91); GO (87); RO (77); MG (75); SC (74); PR (66) e RS (65).

\section{Variações no Td de valor abaixo de 111, de 30.4.21 a 07.5.21.} SP (-3).

AM (7); RO (5); RS (5); MT (1); SC (1); MG (0); PR (-1); GO (-1); TO (-1); MS (-2) e

Para estes onze estados, observamos que apenas os Estados do AM, RO, RS, MT e SC apresentaram variações positivas para os seus $\mathrm{Td}$, sendo que o $\mathrm{AM}$ apresentou novamente o maior aumento de todo país, o que é um fato digno de nota, levando-se em conta a sua condição recente de explosão no número de óbitos como acontece agora em alguns estados. O Estado de MG não apresentou variação no seu Td, Todos os demais estados deste grupo apresentaram variações negativa dos seus números $\mathrm{Td}$, sendo que as maiores diminuições de Td foram para os estados de MS (-2) e SP (-3).

O Estado da BA ocupa o segundo melhor valor (1.237) na média de número de óbitos por milhão de habitantes, deixando o Estado de AL em terceiro lugar, com 1.277. O aspecto positivo é diminuição na queda do Td do Estado do AM, uma vez que o seu Td continuou subindo, com uma variação de sete unidades nesta semana, quando na semana que se encerrou em 12.02.21, a velocidade de queda do Td do AM chegou a -30.

Ressalte-se que no Estado vizinho, o PA, a situação piora tendo este estado perdido posições e, passou a ser o quinto colocado numa comparação entre os Tds, com um Td de 193 dias.

Verifica-se que dos vinte e sete entes da União, oito estados apresentaram diminuições relativas, no número de óbitos, a saber com as suas variações de Td: AM (7), RS (5), RO (5), PE (2), ES (2), MT (1), RR (1) e SC (1).

Oito estados do NE tiveram seu Tds diminuídos, o único estado que não apresentou variação no Td foi PE.

Esclareço que a nossa análise é para os estados como um todo, podendo em algumas capitais estar a situação mais complicada devido ao adensamento populacional.

As médias de crescimentos diários e variações mensais como podem ser observadas no Quadro 1, vão se reduzindo com o aumento do Td.

Um maior Td indica a proximidade do encerramento do crescimento no número de óbitos.

Notar que o número Td maior não está ligado diretamente com os acréscimos no número de óbitos nos últimos 30 dias. O Estado do PE que apresenta o maior Td (263), apresenta uma variação mensal de 1,15, enquanto o Estado de RR com um Td (140) apresenta uma variação mensal de 1,12. O número Td indica, há quantos dias o número de óbitos era a metade do valor atual.

\subsection{Análise das variações mensais no número de óbitos}

Ainda com base nos resultados apresentados no Quadro 1, são analisados, para os dois grupos de estados, como foi a variação no crescimento no número de óbitos em trinta dias até o dia 7 de maio de 2021. 
Variações mensais para os Estados com Td acima da média nacional

ES (1,24); PA (1,24); DF (1,23); CE (1,23); PI (1,22); SE (1,22); RJ $(1,21) ; A C(1,19)$; BA (1,18); MA (1,18); AL $(1,17) ; \operatorname{RN}(1,17) ; A P(1,16) ; \operatorname{PB}(1,16) ; \operatorname{PE}(1,15)$ e RR $(1,12)$.

Pode-se observar que os maiores valores foram: ES, PA, DF e CE.

\section{Variações mensais para os Estados com Td abaixo da média nacional}

MG (1,35); PR (1,30); MS (1,29); SP $(1,26) ; \mathrm{GO}(1,26) ; \mathrm{TO}(1,22) ; \mathrm{MT}(1,21) ; \mathrm{RS}$ $(1,21)$; RO $(1,20)$; SC $(1,20)$ e AM $(1,04)$.

Pode-se observar que os maiores valores foram dos Estados de MG, PR, MS, SP e GO.

Os estados com Td maior que 111 são aqueles que apresentam, neste momento, variações mensais no crescimento entre 1,12 até 1,24.

Os estados com Td menor que 111 são aqueles que apresentam, neste momento, variações mensais no crescimento entre 1,20 e 1,35, sendo AM neste caso a exceção com uma variação de apenas 1,05, mas este estado estará proximamente no bloco com Td acima da média nacional.

\subsection{Número de óbitos dos estados em função do número Td.}

\section{Óbitos diários para os Estados com Td acima da média nacional}

Os estados com Td acima de 111 têm as seguintes participações, na data de 07.5.21, no número diário de óbitos: RJ (257); CE (98); BA (91); PA (62); PE (49); ES (47); PI (36); PB (32); MA (30); DF (30); SE (28); RN (20); AL (18); AC (7); AP (5) e RR (1).

Totalizando um incremento diário de 811 óbitos o que corresponde a $37,5 \%$ do incremento total de 2.165.

Este Grupo representa os estados que estão mais avançados no esgotamento da pandemia e, observa-se que ocorrem reduções relativas no número de óbitos, como é o caso do estado de PE.

\section{Óbitos diários para os Estados com Td, abaixo da média nacional}

Os estados com Td abaixo de 111 apresentam as seguintes participações no número diário de óbitos: SP (583), MG (259), RS (139), PR (130), GO (77), SC (61), MT (30), RO (30), MS (24), TO (12) e AM (9).

Totalizando um incremento diário de 1.354 óbitos o que corresponde a $62,5 \%$ do aumento total de 2.165 .

Os Estados da Região Centro-Oeste, PR, MG, PA, RN, SE e CE vêm aumentando os seus números de óbitos e os seus Tds têm abaixado, o que indica que continuam com taxas de óbitos diários bem acima da média nacional.

Destaque-se que a cidade de Uberlândia iniciou um processo de queda no número de óbitos e, o seu número Td começa a aumentar estando atualmente em 66 , tendo atingido o valor de 47 em 08.4.21, sendo que seu Td em 19.2.21 era 161. O ponto negativo, é que a cidade de Uberlândia apresenta ainda a maior relação de óbitos por milhão de habitantes quando comparado com todas as unidades da federação, apresentando o valor de 3.159 óbitos/M de habitantes.

\section{Observações gerais}

Os estados com Td acima de 111 dias constituem 43,9 \% da população brasileira e, contribuíram com $37,5 \%$ dos óbitos diários, o que indica uma repartição mais favorável para este Grupo. Os seis estados que mais contribuíram na data de 07.5.21, foram os 
seguintes: RJ (257), CE (98), BA (91), PA (62), PE (49) e ES (47), totalizando uma porcentagem de $74,5 \%$.

Os estados com Td abaixo de 111 dias constituem $56,1 \%$ da população brasileira e contribuíram com $62,5 \%$ dos óbitos diários, isto pode ser explicado pela presença neste grupo dos estados mais populosos, SP e MG, além dos estados da Região Sul, todos com altos números de óbitos.

\section{CONSIDERAÇÕES FINAIS}

Tendo em vista o que foi apresentado podemos ainda analisar a respeito do número de óbitos, o que se segue.

O comportamento da curva do número de óbitos para o país, após 413 dias de observação, não se alterou significativamente e, apresentou uma diminuição de 11,7 \% em relação à semana anterior, o que pode ser considerado como uma redução importante no número de óbitos.

As taxas de aumento no número de óbitos permaneceram iguais ou foram superiores por breves períodos, em relação ao que se observou na primeira onda.

O maior aumento diário, na primeira onda, se deu em 29.7.20 com um número de óbitos igual a 1.595 com um acréscimo diário porcentual de 1,77\%.

O maior acréscimo diário, de toda pandemia, ocorreu no dia 06.4.21 com 4.195 óbitos, e que resultou em um aumento diário porcentual de 1,25\%.

Nesta semana a média de crescimento diário atingiu $0,531 \%$, sendo que na semana anterior a média era de 0,626\%.

Neste momento observamos uma queda na média semanal do número de óbitos, porém isto não se dá da mesma forma, como por exemplo, os estados do Sul em comparação com os estados do Nordeste, tendo os primeiros apresentado maiores proporções de quedas no número de óbitos.

Todos estados que apresentam números de Td acima de 111 dias, e que mantêm taxa de óbitos bem abaixo da média nacional, provavelmente atingiram ou estão próximos da "imunidade de rebanho", a saber: PE, AL, MA e SE.

Para comprovar que o aumento no número de óbitos é localizado, podemos resumir a partir de um retrato pontual do dia 07.5.21 que apenas onze unidades da União, divididos em três blocos são responsáveis por 70,3 \% dos 2.165 óbitos, ficando para as demais dezesseis unidades, $29,7 \%$.

Os três blocos são os seguintes: a) os Estados de SP e do RJ são responsáveis de 38,8 $\%$ do número de óbitos. Ao longo do tempo estes dois estados são responsáveis também por cerca de 35\% (ver Quadro 1) do número total de óbitos; b) os Estados do Sul são responsáveis por cerca 15,2\% do número de óbitos. Ao longo do tempo os Estados do Sul são responsáveis por cerca $15 \%$ do número total de óbitos e c) os Estados com taxas superiores (em 07.5.21) à média nacional de acréscimo no número de óbitos, a saber, MG, PI, RO e SE são responsáveis por 16,3 \% do número de óbitos.

Nesta semana que se encerrou na sexta-feira dia 07.5.21, não se observou mais do que 3.000 óbitos diários, contrariando uma tendência, explicitada por Watanabe (2021), de aumento para 5.000 óbitos diários. 


\section{CONCLUSÕES}

Com um abaixamento no número médio diário de óbitos nesta semana de 2.481 para 2.190, a pandemia ainda se prolonga e, depois de quatro semanas, de reduções na média semanal, já é possível admitir que a vacinação começa a surtir efeito.

Contudo, com base no modelo de crescimento exponencial e, com a taxa média diária atual, poderá ser atingido nos próximos 60 dias um número de óbitos superior a 580.000.

Claro que o efeito esperado da vacinação, é que se possa reduzir e, parar este número abaixo dos 550.000 óbitos, mas para isto ocorrer é necessário que a vacinação seja intensificada e os cuidados com o distanciamento e proteção individual continuem a ser mantidos.

\section{REFERÊNCIAS}

BOLETIM - Informe epidemiológico: banco de dados. Disponível em: https://www.uberlandia.mg.gov.br/coronavirus/boletim-municipal-informe-epidemiologico/. Acesso em: 7 mai. 2021.

CONASS Covid-19: banco de dados. Disponível em: http://www.conass.org.br. Acesso em: 7 mai. 2021.

IBGE - cidades e estados. Disponível em: https://www.ibge.gov.br/cidades-eestados.html?. Acesso em: 8 mai. 2021.

LACERDA, Fábio Henrique De Souza. A importância da estatística descritiva na pandemia de Covid-19. Revista Científica Multidisciplinar Núcleo do Conhecimento, Vol. 02, ed. 8, pp. 05-14, 2020.

WATANABE, M. Pesquisa da UFF sobre sazonalidade da transmissão de COVID-19 prevê até $\mathbf{5 0 0 0}$ óbitos diários nos próximos meses. Disponível em: http://www.uff.br/?q=noticias/24-03-2021/pesquisa-da-uff-sobre-sazonalidade-datransmissao-de-covid-19-preve-ate-5000. Acesso em: 8 mai. 2021.

\section{AN ANALYSIS TO FOLLOW THE DEATHS NUMBER CAUSED BY COVID-19 IN BRAZIL}

Abstract: This document presents a study on Covid-19 in the country. The basis is a summary table showing the evolution in the number of deaths. Then, the situations of country, the states and of the city of Uberlandia-MG are discussed, based on parameters such as Duplication Time and the Monthly Variations.

Keywords: Deaths by Covid-19, Coronavirus in Brazil. 